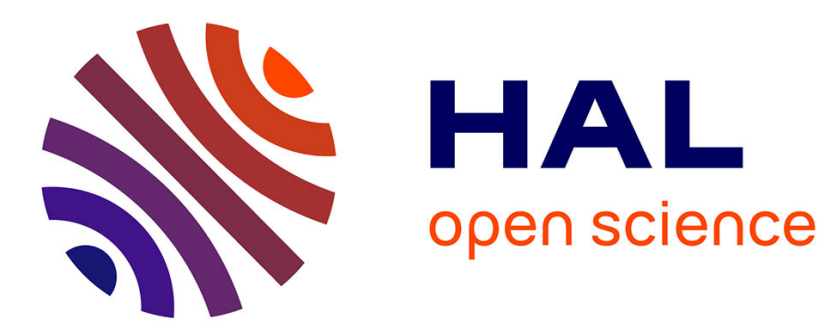

\title{
DJOHA JUIF DANS L'EMPIRE OTTOMAN
}

\author{
Marie-Christine Bornes-Varol
}

\section{To cite this version:}

Marie-Christine Bornes-Varol. DJOHA JUIF DANS L'EMPIRE OTTOMAN. Revue des Mondes Musulmans et de la Méditerranée, 1995, L’humour en Orient (77-78), pp.61-74. hal-02139762

\section{HAL Id: hal-02139762 \\ https://hal-inalco.archives-ouvertes.fr/hal-02139762}

Submitted on 25 May 2019

HAL is a multi-disciplinary open access archive for the deposit and dissemination of scientific research documents, whether they are published or not. The documents may come from teaching and research institutions in France or abroad, or from public or private research centers.
L'archive ouverte pluridisciplinaire HAL, est destinée au dépôt et à la diffusion de documents scientifiques de niveau recherche, publiés ou non, émanant des établissements d'enseignement et de recherche français ou étrangers, des laboratoires publics ou privés. 


\section{DJOHA JUIF DANS L'EMPIRE OTTOMAN}

\section{DJOHA OU HODJA}

\subsection{Les histoires de Djoha dans le répertoire juif}

En Afrique du Nord comme dans l'Empire ottoman les Juifs racontent et transmettent les histoires de Djohá. Elles ont pour eux, en général, un usage très semblable à celui que font de Ch'ha, Goha, Djoha, les populations arabes et de Nasreddin Hodja les Turcs. Parmi les contes judéo-espagnols traditionnels ce que j'appellerai "les contes facétieux de Djoha" occupent une grande place: sur trois volumes de contes judéo-espagnols Matilda Cohen Sarano $\left({ }^{1}\right)$, folkloriste israélienne, en consacre un tout entier à Djoha (1991) qui occupait déjà une section du volume précédent (1986).

Cependant, s'il faut en croire Muriel Klein-Zolty (1991), le personnage de Ch'ha des Juifs d'Afrique du Nord n'a subi aucun processus de judaïsation, contrairement au Djohá judéoespagnol: "Aucune histoire", écrit-elle, "ne dit que Ch'ha est Juif et qu'il va à la synagogue". La judaïsation du personnage serait donc une spécificité judéo-espagnole.

Le phénomène, il faut le signaler demeure restreint et la plus grande partie des histoires de Djohá présentent un héros musulman ou non-marqué. Il n'est indéniablement juif d'après $M$. Cohen Sarano (1994) que dans une trentaine d'histoires.

\subsection{Origine du personnage}

\subsubsection{Le recueil de Salonique}

L'origine du personnage est expliquée en judéo-espagnol (en caractères rachi) par un auteur anonyme qui publie en 1911 à Salonique un recueil d'histoires de Djohá sous le titre $L a$ Vida de Nasreddin Hodja: entièrement assimilé au héros turc, il est présenté comme un personnage véridique, ayant vécu dans la province d'Aksehir; il a été imam à Konya, Ankara et Bursa où, je cite, "il prêchait parmi le peuple la vérité et la justice [...] C'est un sage moralisateur, il donne beaucoup de bons exemples et utilise la ruse pour faire triompher la vérité". Les histoires présentées dans ce recueil sont effectivement des histoires traditionnelles de Nasreddin Hodja mais le héros porte le nom de Djohá.

On y note aussi, par rapport aux exemplaires courants en turc, certaines différences dans la façon de conter. Ainsi, l'histoire de l'énigme de l'oeuf présente en turc Nasreddin comme un

\footnotetext{
1 Le nom de l'auteur est orthographié Koen Sarano dans les ouvrages de 1991 et 1986 mais Cohen Sarano dans l'article de 1994. J'ai conservé cette graphie par souci d'unité.
} 
rusé compère qui donne une réponse stupide à la question stupide de celui qui prétendait le berner, ce qui est précisé: il n'est pas né celui qui pourra se moquer du Hodja. Djoha, en judéoespagnol, fait la réponse "un navet creux fourré de carottes" sans que l'on sache si cela est dû à sa bêtise ou à sa ruse. On pencherait plutôt pour la deuxième solution. Dans toutes les histoires du petit recueil Djohá est musulman.

Dans ce cas, pourquoi le héros ne s'appelle-t-il pas Hodjá, le terme un hodjá existe en effet en judéo-espagnol avec le sens turc et il est couramment employé.

\subsubsection{Sources turques et sources arabes}

Dans son article "Djoha et la Nadirâ", Jean Déjeux ( $C f$. ici-même) montre comme il est difficile de séparer les deux personnages: il apparaît dans l'oeuvre de Djahiz au $\mathrm{IX}^{\circ}$ siècle; il devient le personnage central d'anecdotes recueillies dans un ouvrage anonyme du $\mathrm{X}^{\circ}$ siècle, le Kitab Nawadir Djohá; ce dernier livre a été traduit en turc au $\mathrm{XV}^{\circ}$ et $\mathrm{XVI}^{\circ}$ siècles et grossi du corpus de Nasreddin Hodja pour enfin être traduit en arabe au XVII ${ }^{\circ}$ siècle; les deux héros se sont amalgamés, si bien qu'un recueil arabe de 1880 porte les deux noms (Basset in Mouliéras 1987: 142; Pellat 1965: 605).

\subsubsection{L'anecdote de l'enterrement}

Le personnage de Djoha apparaît dans l'aire ottomane en 1271 dans le Mathnawî de Djelaleddin Rumî, Mewlanâ. Ce grand philosophe soufi écrit en persan mais enseigne la théologie à Konya. Des anecdotes de Djuha servent entre les exemples qui illustrent sa doctrine (Hirt, 1991: 40). Parmi celles-ci l'anecdote de l'enterrement:

"Un enfant pleurait amèrement en se frappant la tête à côté du cercueil de son père, disant: "Pourquoi mon père, t'emporte-t-on pour te placer sous la terre? Ils t'emmènent vers une maison étroite et infecte; il n'y a là ni tapis ni natte; ni lampe la nuit, ni pain le jour; ni odeur ni signe de nourriture ne s'y trouvent, ni porte en bon état, ni accès au toit; pas un seul voisin pour être ton refuge. Ton corps qui était un endroit pour les baisers des gens, comment irait-il dans une maison aveugle et obscure? Une maison sans pitié et une pièce étroite, où ni ton visage ni ta couleur ne demeureront." De cette manière, il énumérait les caractéristiques de la maison, tandis que coulaient de ses yeux des larmes de sang. Djuhî dit à son père: "O mon père vénéré, par dieu ils apportent ce cadavre dans notre maison."" (Djalâl-od-Dîn Rûmî, 1990: 482).

Or cette histoire figure en bonne place dans le premier roman picaresque espagnol La vida de Lazarillo de Tormes (1994: 187). R. Fouché-Delbosc (1900) a remarqué son origine folklorique et sa présence dans le Liber Facetiarum. A. Rumeau en analyse la présence dans

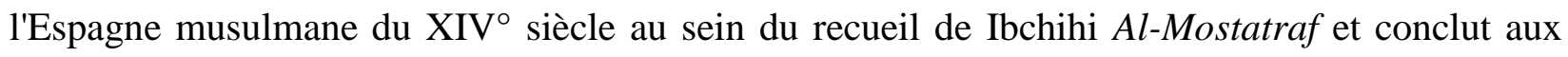
sources arabes de "l'historiette de la casa lóbrega y oscura". Pour finir F. de la Granja (1971: 
235-236) reprend les sources arabes connues de l'historiette sans jamais rencontrer l'allusion à Djoha, la supposant tout de même à partir de la classification de l'anecdote dans un recueil folkloriste allemand consacré au Hodja Nasreddin. L'attribution à Djuhî de l'anecdote du Mathnawî prouve qu'une source arabe a probablement existé, connue et diffusée au Moyen-Age.

Tout ceci nous amène à conclure à une origine espagnole de ce Djoha judéo-espagnol, antérieure à 1492, transportée dans l'Empire ottoman où elle s'est confondue, à l'exception du nom du héros, avec le cycle des anecdotes de Nasreddin Hodja.

\subsection{Caractéristiques du héros}

\subsubsection{Un personnage contradictoire ou controversé}

Les remarques concernant le personnage sont contradictoires. C. Pellat (1965) le décrit comme naïf, simple et quelquefois lourd, mais singulièrement avisé à ses heures (...) il apparaît sous des aspects fort divers.". Ces observations recoupent celles de M. Molho (1960: 120): "Dans les anecdotes contées par les Juifs d'Orient Djoha est en même temps crédule et astucieux. Il est souvent vaincu par ses ennemis mais s'en sort finalement grâce à un geste original ou burlesque qui semble le fait d'un homme ingénu". Ceci concerne l'ensemble du corpus. On retrouve cette opinion exprimée par D. Halbout du Tanney (1990: 5) à propos du héros turc. La même hésitation peut se trouver chez la plupart des auteurs consultés et cités en bibliographie.

Il semble que ces contradictions proviennent de la compilation de sources différentes et de différentes veines d'anecdotes que compliquent encore les traductions turques, persannes et arabes. Cependant le héros arabe, dont le nom est une inversion du terme hodja ainsi que l'a fait remarquer H. V. Sephiha (XX), semble un personnage bien plus négatif que le Hodja turc qui conserve son titre intact. Raison supplémentaire de pencher pour la préexistence d'un corpus d'anecdotes de Djoha parmi les Juifs à leur arrivée dans l'Empire ottoman au $\mathrm{XV}^{\circ}$ siècle.

L'hésitation n'est pas le fait, on l'a vu plus haut, de l'auteur du recueil Salonicien, dont nombre d'histoires infirment pourtant l'introduction. Ce n'est pas non plus celle de H. Batu (s. d.) dans un petit ouvrage intitulé: Nasrettin Hodja - L'humour philosophique".

Alors crédule ou astucieux, ingénu ou rusé, idiot ou philosophe, moraliste ou escroc, qui est Djoha?

\subsubsection{Différents types d'histoires}

S'il est des façons différentes de rapporter la même histoire, on l'a vu avec l'énigme de l'oeuf, il est surtout différent types d'histoires que la confusion des recueils fait coexister. Tant le Hodja turc que le Djohá judéo-espagnol (dans l'ensemble du corpus) se trouvent au centre de trois types principaux d'anecdotes: Djohá escroc, Djohá moralisateur et Djohá naïf et philosophe. 
Comme exemple de la première je donnerai "le clou de Djohá", expression qui désigne la faille d'un contrat dans toute la Méditerrannée. Djohá s'y montre un parfait escroc: il vend sa maison à un prix très bas en exigeant de garder en toute propriété un clou planté dans un des murs. Le client tout heureux accepte la condition dérisoire. Djohá accroche une charogne puante à son clou et le malheureux propriétaire évacue la maison. La marmite de Djohá manifeste le même esprit: il emprunte une marmite au voisin et la rend quelques jours plus tard avec une plus petite en disant "à la bonne heure notre voisin ta marmite a eu un petit". Le voisin prend le tout sans rien dire et ne fait aucune difficulté pour la prêter une nouvelle fois à Djohá qui ne la rend pas. Interrogé celui-ci répond "hélas notre voisin, ta marmite est morte", "Est-ce que ça meurt une marmite?!" répond le voisin outré et Djohá: "eh bien dis donc, ça ne t'a pas autant étonné quand elle a accouché!".

A la veine moralisatrice appartient l'histoire du manteau: de passage dans une ville Djohá demande à être reçu dans la maison du notable du village où a lieu un festin, mais comme il est tout poussiéreux on ne le laisse pas entrer. Il achète alors une belle pelisse et on le place à la droite du maître de maison. Lorsqu'on apporte le potage Djohá y trempe sa pelisse en disant "mange, mange ma pelisse, tout cela c'est pour toi!".

A la veine du benêt philosophe appartient celle de la lanterne: Djohá a perdu sa bague et la cherche à la lumière d'une lanterne de la rue. Un voisin l'interpelle et lui demande ce qu'il a perdu. Se proposant de l'aider, il ajoute "Et où l'as-tu perdue?", "Là-bas, au coin", répond Djohá. "Pourquoi la cherches-tu ici, alors?", dit le voisin. "Moi, je préfère chercher là où il y a de la lumière", répond Djohá.

\subsubsection{Répartition des anecdotes}

Si les histoires sont généralement existantes en turc et en judéo-espagnol on note cependant une nette tendance à faire le départ entre Djohá escroc et Djohá philosophe, Djohá crétin et Djohá moralisateur, dans le corpus judéo-espagnol.

Les contes philosophiques et moralisateurs tendent à quitter le corpus judéo-espagnol de Djohá pour être attribués à Salomon ou au prophète Elie. Ainsi en va-t-il par exemple du conte de la pelisse, attribué à Eliahu ha naví (Cohen Sarano: 1994: 49) et du conte de la chandelle ou Hodja / Salomon donne une bonne leçon à de mauvais sujets qui n'avaient pas respecté les termes d'un pari difficile à tenir.

Par contre on tend à attribuer à Djohá des contes appartenant à d'autres héros archétypiques de l'imbécile ou du crétin; T. Alexander (in Cohen Sarano, 1991: XXIII) remarque ainsi qu'une histoire se rapportant à Dayenu un héros naiff de l'aire culturelle juive se trouve ainsi attribuée à Djohá. Ce glissement de Djohá vers la bêtise fait qu'on le retrouve également héros d'un conte traditionnel juif (commun également dans l'aire ashkénaze) qui rappelle aussi le conte espagnol "el buen cura de Ocaña", la bêtise du héros mise à part. Dans ce conte, le bedeau crétin 
de la synagogue sauve la communauté devant le roi chrétien en mettant en échec ses sages par l'obligation de dire ce que signifie en hébreu ani lo yodea 'je ne le sais pas'. L'histoire pourrait s'arrêter là et le bedeau serait un sage (comme est un sage le serviteur du bon curé d'Ocaña), mais de retour dans sa communauté, pressé de déclarer comment il a eu cette idée de génie, le bedeau répond "cela fait vingt ans que je pose la question à notre rabbin et qu'il me répond qu'il ne sait pas; notre rabbin ne le sait pas et eux ils le sauraient?!". C'est donc par excès de bêtise, de naïveté mais aussi de confiance en son rabbin qu'il a sauvé la communauté, grâce au miracle de la langue sacrée. Cette histoire se trouve rapportée parmi les contes de Djohá dans l'un des recueils cités et je l'ai aussi entendue rapporter comme une histoire de Djohá en Turquie.

Ainsi la tradition judéo-espagnole fait-elle de Djohá plutôt un naïf, un imbécile ou un pauvre homme berné qu'un sage, un philosophe ou un escroc génial.

\section{DJOHA MUSULMAN / DJOHA JUIF DANS LE CORPUS JUDEO-ESPAGNOL}

\subsection{Corpus judéo-espagnol}

Les histoires de Djoha sont transmises oralement et donnent lieu à un grand nombre d'expressions de référence qui en sont tirées. Outre le recueil imprimé de Salonique et le livre de M. Cohen Sarano cités plus haut on trouve des histoires et des expressions dans le dictionnaire de J. Nehama (1977), les contes en français de H. V. Sephiha (1992), éparpillés dans diverses revues judéo-espagnoles

\subsection{Djoha musulman / Djoha non-marqué}

Dans nombre d'histoires relevées dans les sources que je viens de citer, Djoha est musulman, il est imam, il prêche à la mosquée, il porte un turban, sa femme s'appelle Aïche, etc. Dans un plus grand nombre il n'est pas marqué nettement, aucun détail ne permet vraiment de savoir s'il est juif ou musulman. Dans un petit nombre d'histoires enfin il est directement marqué comme juif.

Le départ n'est pas toujours facile à faire car à peine un élément peut avoir varié, ce que remarque également M. Cohen Sarano, qui écarte pour cette raison bon nombre d'histoires dans le corpus qui sert de base à son article sur l'horizon hébraïque de Djoha (1994). Ainsi en va-t-il, par exemple, de l'histoire où Djoha qui a une grande maison est pressé d'accueillir tous les étrangers de passage et il finit par indiquer à l'un de ces invités, particulièrement vorace et parasite, le kal, la synagogue, en lui disant "tiens, c'est la maison du bon Dieu, là on s'occupera de toi". Seul le kal remplace dans cette histoire la mosquée de l'histoire turque et il s'agit peut-être d'une simple adaptation à l'auditoire.

Cependant elle compte au nombre des histoires juives une histoire également connue du 
cycle musulman en intervertissant les places et en donnant le beau rôle (le gâteau mangé) au juif Djoha (1994: 21) là où triomphait Hodja sur deux derviches (Maunoury, 1994: 143).

\subsection{Djoha juif}

\subsubsection{Djoha la victime ou l'inversion de l'anecdote}

Nous avons dit plus haut que Matilda Cohen Sarano (1994) isolait un corpus de 28 histoires dans lesquelles Djoha était indéniablement juif. J'ai puisé plus largement dans son recueil de 1991, dans les récits des folkloristes cités en bibliographie, enfin dans mes propres relevés.

Le point le plus marquant de l'examen de ces histoires est que chaque fois que Djoha est très nettement marqué comme juif il est avant tout un perdant, un malchanceux, comme le shlimazel du folklore ashkénaze.

L'histoire la plus symptômatique de ce point de vue est celle qui présente la situation inverse du conte de la marmite, racontée plus haut. Djoha / Hodja y bernait son voisin, il était en judéo-espagnol non-marqué. Mais un conte inverse existe ou Djoha est marqué comme juif et se trouve être le dindon de la farce. Qu'on en juge : Djoha a acheté une poule pour la kapará 'sacrifice' du jour de Kippur 'le Grand Pardon', il confie la gayinika de kapará à son voisin qui a un poulailler. Quand il prend le lendemain des nouvelles de la poule, le voisin lui donne des oeufs en lui disant "elle va fort bien, d'une poule tu en as trois, et en voici les oeufs" Djoha se réjouit. Le lendemain il lui assure que les trois poules sont devenues neuf et Djoha est de plus en plus content. Vient le jour de Kippur, Djoha réclame sa poule et le voisin lui annonce que les vingtsept poules sont mortes. Devant l'étonnement de Djoha le voisin lui explique que leur mort subite est tout aussi inexplicable que leur multiplication. C'est donc Djoha qui est ici berné.

\subsubsection{Djohá, l'escroc découvert}

Cetee propriété de Djohá juif lui fait attribuer la place du perdant dans un détournement d'un récit talmudique, ainsi que l'a noté M. Cohen Sarano (1994: 5-6). Djohá a parié qu'il ferait perdre patience à son maître (Hillel) qui a la patience d'un saint.

On trouve un conte où Djoha juif est un escroc, mais très rapidement l'histoire se retourne contre lui-même. Djoha loue une suká, une cabane, à une famille pour la fête de Sukot $\left(^{2}\right.$ ). Il la loue à très bas prix à condition d'être invité le soir à boire et à manger. Il mange et boit tellement que la famille préfère lui rendre la cabane, qu'il reloue derechef à d'autres. Mais tout le monde

\footnotetext{
2 Fête des cabanes durant laquelle la famille se réunit pour manger le soir, pendant sept jours, hors de la maison, dans une cabane spécialement dressée à cet effet.
} 
l'apprend bientôt, et se répète ce qui est effectivement une expression judéo-espagnole: kén metyó Djohá en mi suká?, 'qui a mis Djoha dans ma suká?', qui signifie 'que fait cet intrus chez nous?! Jetons-le dehors'. On explique par cette histoire comment l'exclamation des braves gens est devenue proverbiale.

De fait Djoha échoue ici dans son entreprise mais il est aussi celui qui par son attitude associale se met au ban de sa communauté.

\subsubsection{Djohá, le marginal}

De fait de par ses défauts, ses exentricités ou sa bêtise, Djohá se trouve toujours aux marges du groupe. Ceci les proverbes et les expressions traditionnelles l'expriment plus nettement encore que les histoires, puisqu'elles présentent exclusivement une image repoussoir de Djoha, en bute à la critique sociale du groupe, toujours situé à sa limite interne mais menacé d'exclusion. Les proverbes relevés par Nehama (1977) coïncident avec les expressions que j'ai relevées et que consignent K. Perahya, S. De Toledo et alii (1994). On en retrouve certaines dans le relevé de M. Cohen Sarano (1991: 355-379).

Indépendamment de l'expression précédemment citée, on dira de quelqu'un qui se singularise toujours au point d'être tenu à l'écart Djohá ke no kave en la hevrá, 'Djoha qui ne tient pas (qui est de trop) dans l'assemblée'. Un proverbe qui sert à exprimer plaisamment que tout le monde est quitte, dit: Djohá no kere a la malle, ni los mallelís keren a Djohá, 'Djoha n'aime pas les gens du quartier et les gens du quartier n'aiment pas Djoha'. En Yougoslavie on dit d'une personne gênante Djohá en medyo de la sofá, 'Djoha au milieu du sofa' ou encore Djohá no kupo en la suká, 'Djoha n'a pas tenu dans la suka'.

3 Djohá, frontière en matière de tolérance sociale

S'il ne tient pas dans l'assemblée, s'il gêne dans la suká, il encourt donc l'exclusion sociale ou religieuse. De fait, il provoque l'exaspération des autres parce qu'il allie les défauts et le manque de limites. Djoha est toujours au-delà de ce qui est acceptable. Il est donc intéressant de considérer qels sont ces défauts dont l'excès est socialement intolérable.

\subsection{Les défauts de Djoha}

- Il est trop égoïste: Espartyó Djohá, para sí lo más 'Djoha a bien partagé, à lui le plus gros morceau'; Djohá izo una bodika todo para su tripika, 'Djoha a fait une petite fête, et tout pour son petit ventre'.

- Il est versatile: Abolta Djohá detrás de la suká littéralement 'il a tourné Djoha autour de la suka'. On joue ici sur le double sens de aboltar, tourner et changer d'avis, en faisant semblant 
de considérer le sens propre, il est en effet normal de faire le tour de la suka pendant une cérémonie de consécration. Cette expression sert à stigmatiser la versatilité, l'indécision.

- Il est ignorant mais veut parler quand même: Ya favló Djohá, 'Djoha a bien parlé' se dit de quelqu'un qui a dit une ânerie.-

- Il est bavard: 'Djoha est allé au bain/au marché il a trouvé de quoi raconter pendant un an'.

- Il est illogique: devant une attitude inexplicable on dit 'A Djoha on lui a donné du potage, il a éclaté en sanglots'. Il est porteur de sac de toilettes mais il fait porter le sien au bain. Dans le conte des ânes il omet toujours de compter celui sur lequel il est assis. Dans un autre conte pour ne pas que son âne se fatigue il met sa besace à son cou alors qu'il est assis dessus. On lui demande: Djohá ande es tu oreja?, 'Djoha où est ton oreille', il montre la droite avec la main gauche du geste le plus compliqué qui soit, et l'expression se moque de celui qui va chercher midi à quatorze heures. A Jérusalem l'expression por el alma de Djohá", '(faire quelquechose) pour l'âme de Djoha' signifie agir sans raison.

- L'exagération et l'excès marquent tous ses actes: 'Djoha on lui a dit de se moucher il s'est arraché le nez', 'Djoha il a embrassé sa fiancé il lui a sorti un oeil', 'Djoha pour tuer la puce a brûlé le matelas'. Cette dernière expression existe sous une forme neutre en général 'brûler un matelas pour tuer une puce' mais je l'ai trouvé deux fois attribuée à Djoha.

- En affaires il est nul, son travail est inefficace: Ké aze Djohá? aze i dezaze" (Bulgarie) 'que fait Djoha? Il fait et il défait'. 'Djoha achète à dix et revend à neuf' (Turquie), 'Djoha est allé à la place il a perdu au lieu de gagner' (Grèce, Turquie). 'Djoha gagne avec les gens de la maison' (ils le laissent faire) (Bulgarie). Pour quelqu'un qui propose une aide dont on ne veut pas on dit: Ni Djoha kon su ayuda 'pas même avec l'aide de Djoha' avec un possible jeu sur le double sens du mot ayuda 'aide' et 'lavement/clystère', pour dire 'je préfère le faire tout seul'.

- Il anticipe à l'excès: il vend la peau de l'ours avant de l'avoir tué. Beaucoup de proverbes expriment cette idée, le plus caractéristique et le plus courant étant 'Djoha avant de se marier a acheté le berceau, avant de monter à cheval il a commencé à marquer le pas avec les jambes'.

- De nombreux proverbes ou expressions épars font allusion enfin à divers traits négatifs de Djoha : sa paresse, sa brutalité, sa prétention infondée.

\subsection{Djohá, le moins que rien}

Pour ces raisons il est tenu pour un moins que rien: "qui y avait-il à la fête? Djoha et son père" signifie 'trois pelés et un tondu'. Azer pleto por la koltcha de Djohá, 'faire un procès pour le matelas de Djoha', c'est faire un procès pour rien. 'Quand on ne sait pas pourquoi pleurer on pleure pour Djoha qui n'a plus de père' signifie pleurer pour rien, chercher des occasions de pleurer.

- Plus généralement 'faire le Djoha' signifiera 'faire l'imbécile', 'tu es un autre Djoha', 'tu es un 
parfait crétin'. 'Las de Djoha' signifie 'des âneries', Ya sta la savor de Djohá, 'ça a la saveur de Djoha', 'c'est de mauvais goût', etc...

\subsection{Djoha, l'inadapté}

\subsubsection{Inadaptation sociale}

De fait l'inadaptation semble le trait dominant de toutes ces situations, la pire d'entre elles étant l'inadaptation sociale. Djoha agit en effet au mépris des convenances sociales les plus élémentaires:

- 'Djoha s'est habillé: en chabad (endimanché) un jour de semaine', marque l'illogisme, le fait d'agir à contre-temps. 'Tout le monde enterre son père comme il veut Djoha l'a enterré avec la jambe hors de terre' marque son mépris choquant des convenances et une attitude aberrante à partir d'une histoire du recueil général vidée totalement de son contexte malicieux de départ. Il prolonge ses visites au-delà du supportable: Entró Djohá i no salyó, 'Djoha est entré et il n'est pas ressorti' se dit à Monastir des importuns.

- Il ne sait pas distinguer l'essentiel de l'accessoire: 'Djoha la nuit de ses noces a laissé la fiancée / il a laissé ses invités, pour aller donner à manger à l'âne'.

- Il n'a aucun sens du contexte social ou des circonstances: 'Djoha s'est souvenu d'embrasser sa femme le jour où sa mère est morte' et dans une autre proverbe il se souvient d'embrasser sa femme 'le jour de Ticha be $a v^{\prime}$, c'est à dire le jour de deuil qui commémore la destruction du temple.

\subsubsection{Inadaptation au contexte}

L'inadaptation sociale n'est qu'un aspect de l'inadaptation plus générale de Djoha au contexte:

- Il ne prend pas en compte le contexte de référence et interprète toujours à l'envers un signe ambigu: dans un conte, alors qu'il est enfant, sa mère pile du sel, sal, en judéo- espagnol signifie à la fois 'sel et sors!' et il s'obstine à mal interpréter la réponse jusqu'à l'exaspérer.

- Il n'adapte pas le conseil donné en fonction de la situation: Dans un conte la mère de Djoha, présentée comme une pauvre femme accablée par la sottise de son fils, essaie de lui apprendre les bonnes manières: Il faut couper en quatre les boulettes de viande/ ce qui est rond, avant de les manger quand on est invité. La fois suivante Djoha coupe les lentilles en quatre. Comme il est trop chargé en revenant du marché sa mère lui reproche de ne pas avoir engagé un portefaix, la fois suivante il revient du marché avec un portefaix pour porter l'aiguille commandée par sa mère.

- il ne peut passer du concret à l'abstrait et prend tout ce qu'on lui dit au sens propre, au 
pied de la lettre. Dans un grand nombre d'histoires il ne sait pas ce qu'est une tomate (quelquefois une aubergine) et comme on lui a expliqué que c'était rouge avec un petit chapeau vert il apporte un derviche en culotte bouffante rouge et fez vert. On lui demande de 'jeter un oeil de temps en temps sur sa fiancée' le jour de la noce, il lui jette dessus de temps à autre un oeil de boeuf. Comme on l'envoie 'voir si le boucher a des pattes/jambes de veau' il lui retrousse ses chausses et revient en disant que non. Dans un certain nombre d'histoires cette propriété de Djoha est utilisée avec des connotations obscènes.

4. Djohá le malchanceux / Djohá le chanceux

\subsection{Djohá symbole de la malchance}

Au delà des histoires qui le montrent en victime ou en position d'échec, quelques proverbes font apparaître ce trait comme caractéristique de Djoha. Il s'agit presque d'un défaut supplémentaire, il est responsable de sa malchance et il attire la malchance: La estrea/el mazal de Djohá, 'l'étoile/la chance de Djoha' signifie 'quelle déveine!' et servira à prévenir les gens 'Ne comptez pas sur lui, il a la déveine de Djoha', il va tout faire à l'envers.

\subsection{Djohá le simple, Djohá le saint}

Mais les benêts s'ils sont pieux et respectueux reçoivent parfois leur récompense de Dieu même. Car s'il est un domaine où prendre les choses au pied de la lettre est important, en dépit des contraintes du contexte, c'est sans doute le domaine du religieux. Ainsi en va-t-il dans quelques histoires où Djoha juif est récompensé, où les choses tournent à son avantage: Une première fois il distribue tout le bien de ses parents parce que le rabbin a dit que Dieu le rendrait au centuple, il obtiendra ce qu'il cherche quand indigné par les propos des voleurs qui maudissent le dieu des Turcs, celui des Arméniens et celui des Juifs (littéralement 'Et moi je mange le Dieu des Juifs'), il s'écrie "Ah non personne ne mange le Dieu des Juifs parce qu'à moi, il me veut beaucoup de bien". Les voleurs épouvantés s'enfuient en abandonnant leur butin.

Une autre fois à la synagogue au lieu de crier Adonay Ehad, 'Dieu est un', Djoha qui n'y comprend rien hurle de tout son coeur Adonay aher, 'Dieu est autre', aher servant en plus d'euphémisme dans les écrits talmudiques pour désigner ce qui est immonde ou impur y compris la viande de porc. Saisi d'une plainte indignée de ses coreligionnaires, le rabbin tente en vain de lui apprendre la bonne formule. Il l'oublie et doit à plusieurs reprises retourner à Jaffa, où se trouve le rabbin, pour se la faire répéter. Comme le rabbin s'est embarqué, il court sur l'eau lui redemander la formule et parvenu au bateau s'entend évidemment répondre: "Ecoute, dis ce que tu veux comme tu le veux, ta prière vaut plus que celle de tous les autres". Ce conte est également 
répandu dans le monde arabe maghrébin $\left(^{3}\right)$.

Ainsi Djoha réussit par sa sincérité là où les autres échouent par rationnalisme. La confiance dans la lettre en dépit de/ au mépris du contexte lui vaut le succès. Il n'est donc pas étonnant de retrouver Djoha dans le conte traditionnel du folklore juif, à la place de l'habituel bedeau crétin de la synagogue qui sauve la communauté ( $c f$. supra XX).

C'est à la ressemblance de cette histoire avec d'autres anecdotes portant sur des questions difficiles, que l'on doit sans doute de voir Djohá, malin cette fois, triompher du roi dans deux anecdotes inédites, de même origine, rapportées par M. Cohen Sarano (1994: 23-24). Seule la première des deux présente un Djohá juif qui prouve au roi que Jésus n'est pas le fils de Dieu, ainsi que celui-ci l'avait demandé.

Un ou deux proverbes expriment d'autre part l'idée que la chance sourit aux simples d'esprit: 'Djoha l'a jeté à l'envers et il est tombé à l'endroit'.

5. Nouvelles histoires de Djohá

La tendance à attribuer à Djohá la stupidité comme caractère dominant dans l'Empire ottoman explique sans doute qu'on lui attribue à l'heure actuelle des histoires de Mochón de Balat, symbole du Juif du quartier pauvre de Balat qui s'est enrichi et qui a du mal à se conformer à sa nouvelle situation sociale. Ou bien encore il est un benêt qui propose d'abandonner la radio qu'il a emmenée en france parce qu'elle ne sait plus dle turc et que de retour au pays elle ne servira plus à rien.

Matilda Cohen Sarano remarque parallèlement (1994: 7) qu'en Israël on lui attribue une anecdote de Hershele (figure du Juif de la diaspora) dont le ressort est l'irrévérence envers l'hymne national que tous respectent. Il tend à s'assimiler à Chucham le yéménite, personnage d'anecdotes dont elle ne donne pas les caractéristiques, mais qui est l'archétype des derniers immigrés en Israël.

Ces évolutions semblent confirmer Djoha juif dans les caractéristiques évoquées plus haut.

6. conclusion

Lorsque Djoha est Juif, il est un simple d'esprit, un benêt, un inadapté, un malchanceux et un perdant. Il présente de nombreux défauts dont le trait principal est l'inadaptation aux normes sociales. Ce faisant, plus encore dans les proverbes et expressions que dans les anecdotes, il marque les limites du groupe en matière de tolérance sociale et sert de repoussoir aux membres

3 Je remercie Fanny Colonna des renseignements qu'elle m'a fournis sur la petite sainte qui marche sur l'eau dans des circonstances tout à fait semblables. 
de la communauté. Ces histoires coexistent avec les histoires traditionnelles de Nasreddin Hodja, également surnommé Djoha et qui sont elles plus nuancées, Djoha, surtout Djoha musulman, y étant un malicieux, voire un sage utilisant la logique formelle pour faire triompher la vérité, donner des leçons ou berner les autres. Les proverbes et expressions judéo-espagnoles ne retiennent que les aspects négatifs. Ce faisant elles sont plus proches du Ch'ha, Djoha, arabe et donc d'une tradition probablement héritée de l'Espagne avant l'expulsion de 1492.

Il est à noter que si Djoha se situe aux frontières de la communauté et encourt perpétuellement la mise au ban ou le herem, l'exclusion n'est jamais effective, quelque puissante que soit l'exaspération de ses coreligionnaires.XX

\author{
Marie-Christine BORNES-VAROL \\ Etudes hébraïques-INALCO
}

\title{
BIBLIOGRAPHIE
}

Aki Yerushalayim, Moshe Shaul éd., revue de l'émission Kol Israel, Jérusalem.

BASSET, René, Mille et Un Contes, Récits \& Légendes Arabes, Tome I: Contes Merveilleux Contes plaisants, Maisonneuve Frères, Paris, 1924.

BASSET, René, "Recherches sur Si Djoh'a et les anecdotes qui lui sont attribuées", in Les Fourberies de Si Djeh'a (cf. infra: Mouliéras), pp. 141-197.

La Vie de Lazarillo de Tormes, Garnier-Flammarion, 1994. (Edition et introduction de Marcel Bataillon).

BATU, Hamit, Nasrettin Hodja - l'Humour Philosophique, Ajanstürk, Ankara, 1974.

CHANFRAULT, Bernard, "Le corpus de gafsa" in Le Cheval de Troie, 1991, pp. 13-20.

CHANFRAULT, Beernard, "Le personnage de Djoh'a comme figure emblématique de l'humour maghrébin: approche sémiotique" in Humoresques, tome II, Z' éd., Nice, mars 1990, pp. 209-217.

COHEN/KOEN SARANO, Matilda, Sipurei Eliahu Ha-Navi, Jerusalem, 1994. (Bilingue hébreu - judéo-espagnol).

COHEN SARANO, Matilda, "L'orizzonte Ebraico di Giocha", Relazione per il Convegno "Gli 
Orizzonti di Giufa", Noto (Sicilia), 23/24-4-1994. (Communication et corpus de 28 histoires, en italien).

COHEN/KOEN SARANO, Matilda, Djoha Ké Dize? - Kuentos Populares Djudeo-Espanyoles, éd. Kana, Jérusalem, 1991. (Bilingue hébreu - judéo-espagnol)

COHEN/KOEN SARANO, Matilda, Kuentos del Folklor de la Famiya Djudeo-Espanyola, Kana, Jérusalem, 1986. (Bilingue hébreu - judéo-espagnol)

CREWS, Cynthia M., Recherches sur le Judéo-Espagnol dans les Pays Balkaniques, Droz, Paris, 1935.

DEJEUX, Jean, "Avant-propos" in Les Fourberies de Si Djeh'a, (Cf. infra: Mouliéras), pp.7-25.

DELAIS, Jeanne, Les Mille et un Rires de Dj'ha, L'Harmattan, Paris, 1986.

DJALAL-OD-DIN RUMI, Mathnawî - La Quête de l'Absolu, traduit par E. De Vitray Meyerovitch et Djamchid Mortazavi, Editions du Rocher, Paris, 1990.

FENOGLIO, Irène, "Caricature et Représentation du Mythe : Goha" in Images d'Egypte - De la Fresque à la Bande Dessinée, CEDEJ, Le Caire, 1991, pp. 133-143.

FOUCHE-DELBOSC, R.,"Remarques sur Lazarillo de Tormes" in Revue Hispanique, VII, 1900, pp. 94-95.

GALLEY, Micheline et IRAQUI SINACEUR, Zakia, Dyab, Jha, La'âba... - Le Triomphe de la Ruse - Contes marocains du fonds Colin, Classiques Africains, Paris, 1994.

GRANJA (de la), Fernando, "Nuevas Notas a un Episodio del "Lazarillo de Tormes"" in AlAndalus, CSIC, Madrid-Granada, vol. XXXVI, fasc. 1, 1971, pp. 223-337.

GÜNEY, Eflatûn Cem, Nasrettin Hoca Fikralari, Varlik Yayinevi, Istanbul, 1974. (En turc)

HIRT, Jean-Michel, "Le Rire du Soufi" in Le Cheval de Troie, 1991, pp. 37-44.

Le Cheval de Troie - La littérature Orale - Djoh'a - Ch'ha - Giufa - Arabes Juifs et Siciliens, n4, Bordeaux, 1991. 
KLEIN-ZOLTY, Mireille, Contes et Récits Humoristiques du Monde Juif, L'Harmattan, Paris, 1991.

MAUNOURY, Jean-Louis, Sublimes Paroles et Idioties de Nasr Eddin Hodja, Phébus, Paris, 1990.

MAUNOURY, Jean-Louis, Hautes Sottises de Nasr Eddin Hodja, Phébus, Paris, 1994.

MOLHO, Michael, Literatura Sefardita de Oriente, Biblioteca Hebraicoespañola, vol. VII, CSIC, Madrid-Barcelone, 1960.

MOULIERAS, Auguste, Les Fourberies de Si Djeh'a - Contes kabyles, la Boîte à Documents, Paris, 1987.

NEHAMA, Joseph, Dictionnaire du Judéo-Espagnol, CSIC, Madrid, 1977.

NESIN, Aziz, (racontées par), Histoires de Nasreddin Hodja, Traduction française et Introduction de Dominique Halbout du Tanney, Dost Yayinlari, Istanbul, 1990.

PELLAT, Charles, "Djuha" in Encyclopédie de l'Islam, Brill - Maisonneuve \& Larose, Leyde Paris, Tome II, 1965, pp. 605-607.

PERAHYA, Klara, DE TOLEDO, Suzi, DANON, Suzi \& ENDER, Fani, Erensya Sefaradi, Gözlem, Istanbul, 1994.

RUMEAU, Aristide, "Notes au Lazarillo - La casa lóbrega y oscura" in Les Langues Néo-Latines, $\mathrm{n}^{\circ} 172$, fasc. 1, mars-avril 1965, pp. 16-25.

SEPHIHA, Haïm Vidal, Du Miel au Fiel - Contes Judéo-Espagnols, Bibliophane, Paris, 1992.

SEPHIHA, Haïm Vidal, Contes de Djoha, Institut universitaire d'études du judaïsme Martin Buber, Bruxelles, 1981.

SHAUL, Elie, Folklor de los Judios de Turkiya, Isis, Istanbul, 1994.

La vida de Nasreddin Hodja XXX

YÖRENÇ, Kemal, Les plus Belles Anecdotes de Nasreddin Hodja, Aksit, Istanbul, 1994. 


\section{SOURCES}

Mes sources essentielles pour cette recherche ont été les suivantes:

- Un petit recueil en judéo-espagnol (caractères "rachis") publié en 1911 à Salonique sous le titre de . Dans la préface le personnage est présenté comme véridique, ayant vécu dans la province d'Aksehir. Il a été imam à Konya Ankara et Bursa où "il prêchait parmi le peuple la vérité et la justice". C'est un sage moralisateur, ils donnent beaucoup de bons exemples et utilise la ruse pour faire triompher la vérité. Les contes, pour ceux que j'ai pu lire, sont des histoires traditionnelles de Nasreddin Hoca, telles qu'on les trouve dans d'autres recueils en turc. Djoha y est musulman. - Le recueil de contes et proverbes relevé par Matilda Koen Sarrano sous le titre Djoha ké dize?, édition bilingue judéo-espagnol (caractères latins)/ hébreu, éditions Kana, Jérusalem, 1986, entièrement consacré à Djoha, ainsi qu'un chapitre de son recueil de kuentos del folklore de la famiya (ed. Kana 1985).

- Quelques contes originaux publiés en judéo-espagnol dans divers numéros de la revue Aki Yerushalayim, revue de l'émission en judéo-espagnol Kol Israel, dirigée par Moshe Shaul, Jérusalem.

- L'article Djoha, dans le Dictionnaire du judéo-espagnol, de Joseph Nehama, Madrid, CSIC, 1977.

- Le recueil de proverbes Refranes de los judíos sefardíes, de Enrique Saporta y Beja, Barcelone, Ameller, 1978.

- L'anthologie de la littérature judéo-espagnole Le judeo-espagnol, H. V. Sephiha, Paris, ed. Entente, 1986. et

- Quelques contes proverbes et expressions recueillis lors d'entretiens en judéo-espagnol enregistrés dans la communauté juive d'Istanbul.

Notons également qu'il a existé un journal juif Djoha et el Djohaiko, distribué en supplément humoristique par le Journal Israelite de Yezekiel Gabay, en 1860.

\section{DJOHA AUJOURD'HUI}

Dans quelques adaptations modernes Djoha se trouve prendre la place du traditionnel Mochón de Balat, ou Salamón, l'archétype du Juif pauvre et un peu ignorant, qui s'est enrichi brusquement. Une sorte de Marius en Turquie. Les histoires concernant ce personnage moquent son inadaptation à sa nouvelle situation sociale. Dans une histoire rapportée à Izmir Mochon/Djoha qui part en voyage de noces en croisière à Marseille demande à sa femme d'emporter la radio pour avoir des nouvelles de Turquie. Au moment du retour il lui conseille de 
la laisser à l'hôtel parcequ'elle a oublié le turc, elle ne sait plus que le français elle ne pourra donc pas leur servir en Turquie. 